\section{BMJ Open Respiratory Research}

\title{
Evaluation of 'care bundles' for patients with chronic obstructive pulmonary disease (COPD): a multisite study in the UK
}

Katherine Morton, ${ }^{1}$ Stephanie MacNeill, ${ }^{2}$ Emily Sanderson, ${ }^{2}$ Padraig Dixon, ${ }^{2}$ Anna King, ${ }^{2}$ Sue Jenkins, ${ }^{3}$ Chris Metcalfe, ${ }^{2}$ Ali Shaw, ${ }^{2}$ Melanie Chalder, ${ }^{2}$ Jonathan Benger, ${ }^{\oplus, 5}$ William Hollingworth, ${ }^{2}$ James Calvert, ${ }^{6}$ Sarah Purdy ${ }^{\oplus}$

\section{ABSTRACT}

Background Chronic obstructive pulmonary disease (COPD) accounts for $10 \%$ of emergency hospital admissions in the UK annually. Nearly $33 \%$ of patients are readmitted within 28 days of discharge. We evaluated the effectiveness of implementing standardised packages of care called 'care bundles' on COPD readmission, emergency department (ED) attendance, mortality, costs and process of care.

Methods This is a mixed-methods, controlled before-andafter study with nested case studies. 31 acute hospitals in England and Wales which introduced COPD care bundles (implementation sites) or provided usual care (comparator sites) were recruited and provided monthly aggregate data. 14 sites provided additional individual patient data. Participants were adults admitted with an acute exacerbation of COPD.

Results There was no evidence that care bundles reduced 28-day COPD readmission rates: $\mathrm{OR}=1.02(95 \% \mathrm{Cl} 0.83$ to 1.26). However, the rate of ED attendance was reduced in implementation sites over and above that in comparator sites (implementation: IRR=0.63 ( $95 \% \mathrm{Cl} 0.56$ to 0.71 ); comparator: IRR=1.12 (95\% Cl 1.02 to 1.24$)$; group-time interaction $\mathrm{p}<0.001$ ). At implementation sites, delivery of all bundle elements was higher but was only achieved in $2.2 \%$ (admissions bundle) and $7.6 \%$ (discharge bundle) of cases. There was no evidence of cost-effectiveness. Staff viewed bundles positively, believing they help standardise practice and facilitate communication between clinicians. However, they lacked skills in change management, leading to inconsistent implementation.

Discussion COPD care bundles were not effectively implemented in this study. They were associated with a reduced number of subsequent ED attendances, but not with change in readmissions, mortality or reduced costs. This is unsurprising given the low level of bundle uptake in implementation sites, and it remains to be determined if COPD care bundles affect patient care and outcomes when they are effectively implemented.

Trial registration number ISRCTN13022442.

\section{INTRODUCTION}

Chronic obstructive pulmonary disease (COPD) is one of the most common

\section{Key messages}

Systematic review evidence from a small number of randomised controlled trials (RCTs) suggested that discharge bundles for patients with chronic obstructive pulmonary disease (COPD) lead to fewer readmissions but do not significantly improve mortality or quality of life. Few data are available on admission bundles for COPD.

- This study found that admission and discharge care bundles for COPD had little impact on patient outcomes, including readmissions and mortality or healthcare costs, but appeared to be associated with a reduced number of subsequent attendances at the emergency department.

- Evaluation of 'real world' implementation of admission and discharge care bundles highlighted the challenge of effective implementation of COPD bundles and does not replicate the effectiveness of implementing care bundles demonstrated in RCTs.

respiratory diseases in the $\mathrm{UK}$, with an estimated prevalence of 1.2 million people. ${ }^{1}$ Globally, the estimated prevalence of COPD was 251 million cases in 2016, and it is estimated that 3.17 million deaths were caused by the disease in 2015, which is $5 \%$ of all deaths. $^{2}$ COPD accounts for $10 \%$ of emergency hospital admissions in the UK, and the number of admissions has increased by $50 \%$ in the last decade. ${ }^{13} \mathrm{~A}$ third of these patients are readmitted within 28 days of discharge. ${ }^{3}$ COPD admissions are estimated to cost the National Health Service (NHS) £491 million per year.

The care provided for COPD varies across European countries, and an audit in the NHS highlighted wide variation in treatment provision and outcomes for patients admitted for COPD. ${ }^{45}$ This disparity was particularly marked in relation to mortality. It also showed that a significant proportion of the observed 
variability could be explained by access to expert care and evidence-based interventions. There is, therefore, opportunity to improve outcomes for patients with COPD by ensuring that care is consistently provided to a high standard.

The Institute for Healthcare Improvement developed the concept of care bundles with the aim of improving care processes to the highest levels of reliability, which would result in improved outcomes. ${ }^{6}$ A care bundle can be defined as a set of evidence-based clinical interventions or actions which when performed reliably improve patient outcomes. Admission and discharge care bundles for COPD were developed by the British Thoracic Society (BTS) in association with NHS Improvement. ${ }^{7}$ The aim of the admission care bundle is to reduce in-hospital mortality and length of stay, and the discharge care bundle was designed to reduce readmissions. Early pilots in the UK prior to this study suggested possible benefits from care bundles in COPD and other conditions. ${ }^{89} \mathrm{~A}$ single site evaluation of COPD admission care bundles showed delivery of care was improved in the emergency department (ED).$^{10}$ Further studies and a systematic review have focused on discharge bundles, suggesting a reduction in readmissions but highlighting that the impact of care bundles on processes and outcomes of care is poorly understood. ${ }^{11-14}$

This study evaluated the effectiveness of introducing admission and discharge care bundles for patients with an acute exacerbation of COPD (AECOPD) as a means of improving hospital care, and reducing readmissions and mortality, and explored the impact on cost of care and patient and staff experience.

\section{METHODS}

Design

We conducted a mixed-method evaluation using a controlled before-and-after design with nested qualitative case studies to examine the effect of implementing care bundles (online supplementary figure 1). The study was conducted between 2014 and 2017. More details about the study methodology can be found in the published protocol paper. ${ }^{15}$

\section{Setting and sampling}

We recruited 31 acute hospitals in England and Wales to the study, approaching sites via the BTS and the National Institute for Health Research Clinical Research Network. This included 19 sites which either introduced care bundles during the study period or used care bundles routinely in COPD care (implementation sites), and 12 sites which provided 'usual care' throughout the study period (comparator sites). An 'index date' was defined for all sites based on when they implemented the bundlesin the case of implementation sites-or the implementation date of a similar site in the comparator sites. Application of a quality improvement (QI) methodology was encouraged in implementation sites throughout the duration of the study using video-conferencing, face-toface training sessions and one-to-one mentoring. ${ }^{7}$

A subset of these sites provided pseudoanonymised details of all individual patient-level admissions over a period of 12 months preindex and postindex date. With seven pairs of similar implementation and comparator sites, it was estimated that there would be a sample of approximately 10000 admissions per year. Assuming $30 \%$ of patients were readmitted in comparator sites, this would allow us to detect a $9 \%$ absolute difference in the COPD readmission rate at 28 days with $90 \%$ power and $5 \%$ significance level.

\section{Data collection}

Each site was asked to provide data covering a 24-month period-12 months prior to their implementation index date and 12 months afterwards. All sites provided monthly aggregated, routinely collected, Trust-level data for this period (level 1 data). The data included COPD admissions rates, COPD readmission at 28 days and 90 days, mortality, length of stay, ED attendances, and delivery of bundle elements where appropriate. COPD admissions were identified as those with International Classification of Diseases 10th Revision codes J41-44 in the first episode of care. $^{7}$

Sites providing pseudoanonymised patient-level data (level 2) reported outcomes, sociodemographic, clinical and procedure codes plus the information gathered for level 1 sites. Demographic data on individual patients allowed us to study the characteristics of patients admitted with AECOPD and adjust for these in analyses. Each level 2 site was also asked to provide details from the case notes of a random sample of 140 postindex date COPD admissions to provide data on the delivery of the various components of the care bundles $(n=2240)$. Quantitative data submitted to the research team were compiled and checked for both validity and consistency.

Qualitative data were collected from six level 3 sites about the process of bundle implementation or usual care. In addition, data on the context in which bundles were delivered, and the impact on staff, patients and carers, were collected by KM and AK. Methods used to capture data at these four implementation and two comparator sites included document analysis, non-participant observation of patient care, and indepth interviews with health professionals, patients and carers following both admission and discharge, guided by topic guides and observation schedules.

\section{Care bundle components}

The following were the components of the BTS admission bundle:

- A correct diagnosis of AECOPD to be confirmed.

- An oxygen assessment should be undertaken and the correct target range prescribed within $30 \mathrm{~min}$.

- Recognise and respond to respiratory acidosis within 1 hour of admission. 
- Medication (steroids and nebulisers) to be administered within 4 hours of admission.

- Review by respiratory team to take place within 24 hours of admission.

The following were the components of the BTS discharge bundle:

- Patients should have respiratory medications and inhaler technique assessed prior to discharge.

- All patients should receive a written plan for how to manage a further AECOPD and should receive a discharge pack of 'emergency' drugs prior to discharge.

- Smoking status should be assessed together with a willingness to quit, and for those patients indicating a wish for further assistance a referral should be made to a stop smoking programme.

- All patients should be assessed for their suitability for pulmonary rehabilitation prior to discharge.

- Community follow-up within 2 weeks of discharge from hospital should be organised.

\section{Data analysis}

Quantitative analysis of effectiveness data

Trust-level aggregate outcomes were reported monthly for the 12 months before and after their index date. These monthly data were used to calculate the mean change of all outcome measures postindex date at each site. This mean change was then compared between implementation and comparator sites using linear regression analyses on monthly outcomes to estimate how the change postindex date differed between implementation and comparator sites after adjustment for the number of COPD admissions, overall 28-day readmission rate and in-hospital mortality rates in the preindex date period.

The characteristics of patients having at least one COPD admission in the preindex date period were compared between implementation and comparison sites. Linear, logistic and ordered logistic regression models with SEs adjusted for Trust-level clustering were used to test for differences between groups.

Individual-level patient outcomes were studied using a range of multilevel regression models to compare the change in outcomes postindex date using an indicator variable (preindex or postindex date) between the implementation and comparator sites. These analyses controlled for clustering within observations from the same patient and within each Trust using random effects for patient and Trust. Multilevel logistic regression models were used for binary outcomes. Where logistic regression failed to converge, multilevel Poisson models with robust SEs were used. Multilevel negative binomial regression models were used for length of stay and number of emergency admissions due to a large variance in outcome. Models were first run without adjustment for potential confounding, then rerun adjusting for age, sex, ethnicity and Index of Multiple Deprivation quintile. For each logistic regression analysis, ORs and 95\% CIs were presented, while for negative binomial and Poisson models the incidence rate ratios (IRRs) and 95\% CIs were presented. No formal adjustment was made for multiple testing. Likelihood ratio tests (or Wald tests where robust SEs were used) were used to test for the presence of group-time interaction.

The extent to which sites implemented each element of the bundle was recorded using case note extraction data, and the results were summarised by group using frequencies and proportions. $\chi^{2}$ tests were used to compare the proportion of patients receiving bundle elements between implementation and comparator sites.

\section{Qualitative analysis}

Interviews with staff, patients and carers and observational data from level 3 sites were collected, anonymised and uploaded into NVivo. ${ }^{16}$ All qualitative data were examined using cross-case thematic analysis and analysed both inductively and deductively. ${ }^{17}$ The analysis sought to identify similarities and differences between sites, highlighting aspects which might be transferable to other hospitals intending to implement care bundles. Attention was also given to overlaps or divergence between aspects of practice observed.

\section{Quantitative analysis of cost-effectiveness data}

An economic evaluation considering the 90-day period following the index admission for COPD was undertaken in level 2 sites. We estimated per-patient secondary care NHS costs using healthcare resource group unit costing methodology, ${ }^{18}$ where patient-specific resource use was valued using nationally representative sources, for example, NHS reference costs, ${ }^{19}$ the British National Formulary, ${ }^{20}$ and Unit Costs of Health and Social Care. ${ }^{21}$ In the absence of individual-level data on self-reported quality of life or other outcome measures, 90-day mortality following the index admission served as the main outcome measure for the cost-effectiveness analysis. This permits the incremental costs associated with care bundles to be associated with incremental deaths avoided. Cost-effectiveness was calculated as a ratio of the difference in NHS secondary care costs between intervention and comparator sites to the between-site differences in 90-day mortality. Detailed methods are described in the protocol paper ${ }^{15}$ and study report. ${ }^{22}$

\section{Patient and public involvement}

Throughout the 40-month study, the research team conducted a range of patient and public involvement (PPI) activities to ensure that the protocol was properly implemented and that any findings were appropriately interpreted in the light of patient and carer experience. Patients and carers were involved in suggesting the original idea for the study, then commenting on the application for funding, including aims and objectives, methods and PPI. An active PPI group was then formed which 
Table 1 Trust-level outcomes (monthly) pooled across implementation and comparator sites

\begin{tabular}{|c|c|c|c|c|c|}
\hline Outcome & Group & Preindex & Postindex & $\begin{array}{l}\text { Difference in the change } \\
\text { postindex date between } \\
\text { implementation and } \\
\text { comparator sites* }(95 \% \mathrm{Cl})\end{array}$ & $P$ value \\
\hline \multirow{2}{*}{$\begin{array}{l}\text { Number of COPD admissions, } \\
\text { mean (SD) }\end{array}$} & Comparator & $48.02(21.91)$ & $49.33(19.33)$ & \multirow[t]{2}{*}{$0.17(-6.57$ to 6.90$)$} & \multirow[t]{2}{*}{0.960} \\
\hline & Implementation & $52.49(18.21)$ & $53.93(17.94)$ & & \\
\hline \multirow{2}{*}{$\begin{array}{l}\text { 28-day COPD readmission rate, } \\
\text { mean (SD) }\end{array}$} & Comparator & $11.49(3.60)$ & $12.79(4.36)$ & \multirow[t]{2}{*}{$-1.31(-5.37$ to 2.75$)$} & \multirow[t]{2}{*}{0.513} \\
\hline & Implementation & $15.95(9.20)$ & $16.07(11.53)$ & & \\
\hline \multirow{2}{*}{$\begin{array}{l}\text { 28-day overall readmission rate, } \\
\text { mean (SD) }\end{array}$} & Comparator & $23.63(6.70)$ & $24.91(7.46)$ & \multirow[t]{2}{*}{$-1.17(-4.51$ to 2.17$)$} & \multirow[t]{2}{*}{0.478} \\
\hline & Implementation & $23.05(9.90)$ & $23.10(9.96)$ & & \\
\hline \multirow{2}{*}{$\begin{array}{l}\text { 90-day COPD readmission rate, } \\
\text { mean (SD) }\end{array}$} & Comparator & $22.35(5.59)$ & $23.12(7.27)$ & \multirow[t]{2}{*}{$-4.00(-8.87$ to 0.87$)$} & \multirow[t]{2}{*}{0.103} \\
\hline & Implementation & $25.47(16.42)$ & $22.38(11.92)$ & & \\
\hline \multirow{2}{*}{$\begin{array}{l}\text { Number of ED attendances for } \\
\text { COPD per month, mean (SD) }\end{array}$} & Comparator & $32.45(25.17)$ & 37.60 (26.69) & \multirow[t]{2}{*}{$-3.38(-14.59$ to 7.82$)$} & \multirow[t]{2}{*}{0.525} \\
\hline & Implementation & $45.87(39.27)$ & $47.03(38.22)$ & & \\
\hline \multirow[t]{2}{*}{ Length of stay, mean (SD) } & Comparator & $6.21(1.96)$ & $5.95(1.60)$ & \multirow[t]{2}{*}{$-0.30(-1.10$ to 0.51$)$} & \multirow[t]{2}{*}{0.453} \\
\hline & Implementation & $6.76(1.36)$ & $6.16(1.17)$ & & \\
\hline \multirow[t]{2}{*}{ Total bed days, mean (SD) } & Comparator & $288.48(156.30)$ & $275.86(115.58)$ & \multirow[t]{2}{*}{$7.83(-53.66$ to 69.32$)$} & \multirow[t]{2}{*}{0.795} \\
\hline & Implementation & $333.15(121.35)$ & 326.37 (136.62) & & \\
\hline
\end{tabular}

${ }^{*}$ Adjusted for the number of COPD admissions, in-hospital mortality and overall 28-day readmission for COPD in the preindex date period. COPD, chronic obstructive pulmonary disease; ED, emergency department.

continued to provide input on recruitment and patient burden during data collection and study documentation such as consent forms and information sheets. PPI participants also provided feedback on the data available from both qualitative and quantitative sources, commenting on the extent to which it validated their own experiences of care, and provided feedback on possible approaches to the dissemination of the results of the study which would inform patient groups and the wider community.

\section{RESULTS}

\section{Quantitative findings}

Analysis of Trust-level aggregate data

Nineteen sites implementing COPD care bundles and 11 comparator sites provided preindex and postindex date data for analysis. One other comparator site was unable to provide data for the full study period. Pooled results are presented in table 1 .

Implementation sites had a slightly higher mean number of monthly COPD admissions during the preindex date period (52.5 vs 48.0) and a slightly higher mean monthly readmission rate for COPD $(15.2 \%$ vs $11.5 \%)$.

When comparing outcomes for readmissions, ED attendances, length of stay and bed days, the change observed between the preindex and postindex date periods in the implementation sites did not differ from that seen in the comparator sites (table 1). Graphs displaying the monthly 28-day readmission rates for COPD (preindex date) over time by site showed no obvious trends for most sides, although for some there was a clear, strong trend of an increasing admission rate over the year (online supplementary figure 2).

\section{Analysis of individual patient-level data}

During the preindex date period, patients in the implementation sites tended to be slightly younger, although the evidence of difference was weak (table 2).

There were 19097 emergency hospital admissions for COPD during the full study period, of which $13.0 \%$ resulted in a readmission for COPD within 28 days. In the preindex date period, $11.6 \%$ of COPD admissions had a readmission within 28 days in implementation sites. In comparator sites, the proportion was $14.7 \%$. Postindex date, these proportions were $10.8 \%$ in implementation sites but remained at $14.7 \%$ in comparator sites. In multivariable regression analyses, there was no evidence that the 28-day readmission rates changed postindex date in either the implementation or comparator sites, and there was no difference in the changes between these two groups (OR for group-time interaction term $=1.02$ (95\% CI 0.83 to 1.26 ); $\mathrm{p}=0.865$ ) (table 3 ).

Similar findings were observed for 90-day COPD readmission rates and 90-day mortality (table 3 ). In the case of 28-day all-cause readmissions, there was weak evidence of a reduction in the implementation sites $(\mathrm{OR}=0.88(95 \%$ CI 0.77 to 1.00$)$ ), but little evidence that this reduction differed from changes observed in the comparator sites (group-time interaction term $\mathrm{OR}=0.88$ (95\% CI 0.74 to 1.05); $\mathrm{p}=0.156$ ) in analyses adjusting for confounders. There was only weak evidence that the reduction in the length of stay in the implementation sites differed from that observed in the comparator sites (group-time interaction term $\mathrm{OR}=0.94$ (95\% CI 0.88 to 1.01$) ; \mathrm{p}=0.100)$.

Changes in in-hospital mortality rates did not differ between comparator sites and implementation sites (group-time interaction term OR=1.05 (95\% CI 0.77 to 
Table 2 Characteristics of patients having at least one COPD admission in level 2 implementation and comparator sites at preindex date

\begin{tabular}{|c|c|c|c|}
\hline Characteristics & Implementation sites & Comparator sites & $P$ value \\
\hline Number of COPD admissions & 4657 & 4515 & \\
\hline Number of patients having at least one COPD admission & 2732 & 2549 & \\
\hline Age, mean (SD) & $71.92(12.08)$ & $73.02(11.45)$ & 0.166 \\
\hline Sex, number of male (\%) & $1351(49.5)$ & $1240(48.7)$ & 0.785 \\
\hline \multicolumn{4}{|l|}{ Ethnicity, number of patients (\%) } \\
\hline White & $2305(85.59)$ & $2195(87.7)$ & 0.748 \\
\hline Other & $388(14.4)$ & 307 (12.3) & \\
\hline Comorbidity, mean Charlson score (SD) & $2.07(1.58)$ & $2.14(1.65)$ & 0.673 \\
\hline \multicolumn{4}{|l|}{ Socioeconomic status, number of patients per IMD quintile (\%) } \\
\hline 1 (most deprived) & $103(37.0)$ & $930(38.2)$ & 0.763 \\
\hline 2 & 552 (20.4) & 554 (22.8) & \\
\hline 3 & $493(18.2)$ & $506(20.8)$ & \\
\hline 4 & $339(12.5)$ & $244(10.0)$ & \\
\hline 5 (least deprived) & $326(12.0)$ & $198(8.1)$ & \\
\hline
\end{tabular}

COPD, chronic obstructive pulmonary disease; IMD, Index of Multiple Deprivation.

1.44); $\mathrm{p}=0.741)$. The number of ED attendances after an initial emergency admission for COPD increased postindex date in the comparator sites (IRR $=0.78(95 \%$ CI 0.63 to 0.97$)$ ) while the rates dropped in the implementation sites (IRR $=0.63$ (95\% CI 0.56 to 0.71$)$ ), and there was evidence that the drop was greater in the implementation sites (group-time interaction term IRR $=0.56$ (95\% CI 0.48 to 0.65$) ; \mathrm{p}<0.001)$.

Sites provided data on care bundle delivery for 1525 patients (77.8\% of those requested) (table 4). Delivery of bundle elements generally occurred more frequently in implementation sites than in comparator sites, although few patients in implementation sites received all five BTS-specified bundle elements (admissions bundle: 2.2\%; discharge bundle: $7.6 \%$ in implementation sites). The average number of admission bundle elements received in comparator sites was $2.2(\mathrm{SD}=1.1)$ vs $2.6(\mathrm{SD}=1.1)$ in implementation sites. The average number of individual care elements received on discharge in comparator sites was $1.8(\mathrm{SD}=1.3)$ vs $2.8(\mathrm{SD}=1.7)$ in implementation sites (tables 4 and 5).

\section{Qualitative findings}

Staff perceptions of care bundles were largely positive. Bundles were described as useful for standardising working practices, supporting a clear patient care pathway, facilitating communication between different teams and identifying support required by patients following discharge from hospital. Care bundles were also perceived by staff as a means for embedding reliable, sustainable QI. Staff highlighted the need for managerial support, resourcing and regular training to facilitate QI. and we know it's always been done. (IMP03 ACU6, Lead Nurse, Acute Care)

with a care bundle, there is a better chance they are going to go out on the right treatment really, particularly if they have not been under the Respiratory Team, and they will have access to more services. (IMP11 ACU7, ED Consultant) I think when patients get discharged I think our checklist that we have works really, really well because it's a good sort of pointer for us to try and get patients in to see the appropriate people. (COMP06 ACU3, Respiratory Nurse)

Patients and carers highlighted the need for specialist care and support at the point of discharge, as well as timely follow-up in the community.

I think I'll be quite happy and contented as long as I know I'll be under the COPD nurses. (IMP05 PAT7, Patient)

The staff data echoed this, by highlighting how pressure around patient numbers, resourcing and staffing in the current context of the NHS can mean that it is not always possible for patients to receive as thorough care, particularly in relation to follow-up, as acute and community staff would prefer. Discharge bundles created opportunities to discuss available services and potential management options.

\section{Cost-effectiveness findings}

COPD care bundles were very unlikely (probability $<0.01)$ to be cost-effective at an arbitrary cost-effectiveness threshold of $£ 20000$ per death avoided at 90 days in a fully adjusted, multiply imputed economic model. 


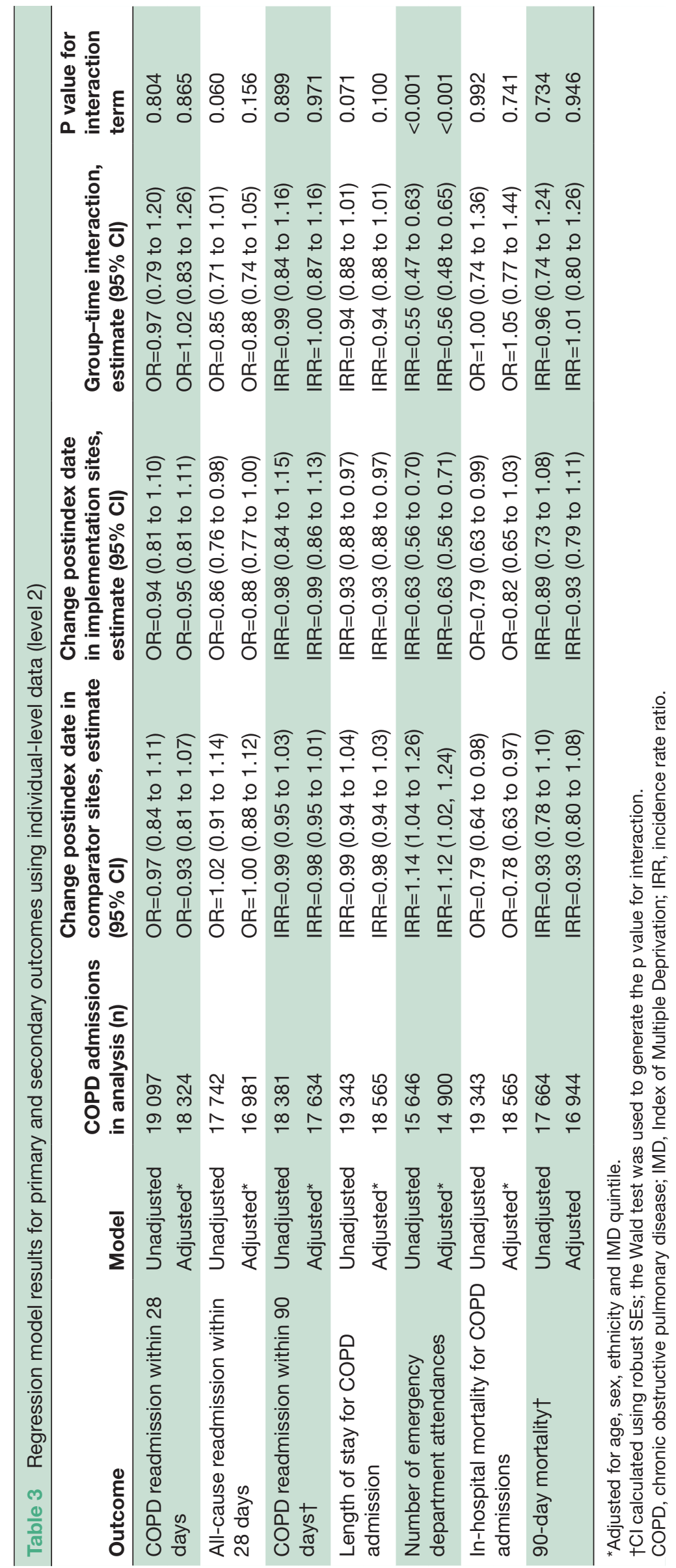


Table 4 Delivery of individual elements of the admissions bundle at level 2 comparator and implementation sites

\section{Admissions bundle}

\begin{tabular}{|c|c|c|c|c|}
\hline Bundle element & Delivery & $\begin{array}{l}\text { Comparator } \\
\text { sites, } \mathrm{n}(\%)\end{array}$ & $\begin{array}{l}\text { Implementation } \\
\text { sites, } \mathbf{n}(\%)\end{array}$ & P value \\
\hline \multicolumn{5}{|l|}{ 1. Correct diagnosis of AECOPD. } \\
\hline 1b. ECG result within 4 hours. & Yes & $473(74.8)$ & $656(83.5)$ & $<0.001$ \\
\hline $1 a$ and $1 b$ & Yes & $350(59.9)$ & $382(66.4)$ & 0.022 \\
\hline $\begin{array}{l}2 \mathrm{~b} \text {. When } \mathrm{pH} \text { is less than } 7.35 \text {, assess suitability for Non-invasive } \\
\text { ventilation (NIV) and implement within } 3 \text { hours of admission. }\end{array}$ & Yes & $345(94.5)$ & 492 (98.0) & 0.006 \\
\hline $2 a$ and $2 b$ & Yes & $193(57.4)$ & $283(61.9)$ & 0.203 \\
\hline \multicolumn{5}{|l|}{ 4. Correct prescription of medication for AECOPD at admission. } \\
\hline $\begin{array}{l}\text { 4a. Steroids prescribed and administered within } 4 \text { hours of } \\
\text { admission when necessary. }\end{array}$ & Yes & 479 (80.6) & 496 (70.2) & $<0.001$ \\
\hline $\begin{array}{l}\text { 4b. Antibiotics prescribed and administered within } 4 \text { hours of } \\
\text { admission when necessary. }\end{array}$ & Yes & 445 (73.4) & 535 (74.6) & 0.624 \\
\hline $\begin{array}{l}\text { 4c. Nebulisers prescribed and administered within } 1 \text { hour of } \\
\text { admission when necessary. }\end{array}$ & Yes & 327 (52.2) & 315 (43.5) & 0.002 \\
\hline $4 a, 4 b$ and $4 c$ & Yes & $214(35.8)$ & 207 (29.5) & 0.015 \\
\hline \multicolumn{5}{|c|}{ 5. Review by respiratory specialist (specialist nurse, doctor or physiotherapist) within 24 hours. } \\
\hline & Yes & $100(17.4)$ & $274(39.3)$ & $<0.001$ \\
\hline
\end{tabular}

AECOPD, acute exacerbation of chronic obstructive pulmonary disease.

\section{DISCUSSION}

We found no evidence that change in readmissions over time differed between implementation and comparator sites. Furthermore, no difference was observed for in-hospital mortality, length of stay or 90-day readmission. There was a reduction in ED attendances for those receiving care in implementation sites. Additionally, there was a small reduction in the duration of hospital stay for patients in the implementation group. The implementation of care bundles was unlikely to be cost-effective from a secondary care perspective.

Qualitative data suggested that health professionals valued bundles as a means of focusing on standardised quality of care, but staff lacked experience and knowledge in QI strategies. This reflects the findings of Lennox et $a l,{ }^{23}$ who identify the barriers and facilitators to care bundle implementation. As other authors have identified, the local context, governance structures and financial incentives for support were crucial in determining whether, and how effectively, sites implemented care bundles. ${ }^{24}$ We observed the use of discharge 'checklists' at comparator sites, which often represented 'bundling by another name'. As a result, similar processes and activities seemed to be taking place at implementation and comparator sites. This is likely to have reduced the ability of the study to demonstrate difference in outcomes attributable to care bundles.

To achieve improvements in outcomes, care bundles must meet three criteria: the target outcome must be sensitive to change and responsive to the elements within the bundle; the care bundles must be reliably implemented to ensure that the majority of patients receive bundle-led care; and use of the care bundle must improve process reliability. ${ }^{13}$ In this study the bundles were not delivered reliably, with delivery of the full fiveitem admission bundle and discharge bundle being very low, although there is some evidence from our data that those patients in receipt of a bundle received more of the required elements of care, suggesting some improvement in process reliability. The third criterion was not met since most patients did not receive the full set of bundle elements. Therefore, effective implementation of the admission and discharge bundles did not occur and the anticipated difference between groups was not observed.

The original BTS COPD care bundles project included QI methodology and continuous data collection to ensure compliance with elements of bundle delivery. ${ }^{713}$ However, few sites fully engaged with this process during this study, and it was clear from site visits 


\begin{tabular}{|c|c|c|c|c|}
\hline \multicolumn{5}{|l|}{ Discharge bundle } \\
\hline Bundle element & Delivery & $\begin{array}{l}\text { Comparator } \\
\text { sites, } \mathrm{n}(\%)\end{array}$ & $\begin{array}{l}\text { Implementation } \\
\text { sites, } \mathbf{n}(\%)\end{array}$ & $P$ value \\
\hline \multicolumn{5}{|l|}{ 1. Assess respiratory medications and inhaler technique. } \\
\hline 1a. Respiratory medications. & Yes & $342(53.4)$ & $542(68.5)$ & $<0.001$ \\
\hline 1b. Inhaler technique. & Yes & $106(17.1)$ & $307(40.4)$ & $<0.001$ \\
\hline $1 a$ and $1 b$ & Yes & $102(16.1)$ & $302(39.4)$ & $<0.001$ \\
\hline \multicolumn{5}{|l|}{ 2. All patients should receive } \\
\hline 2a. Written pack about managing further AECOPD. & Yes/not applicable & $253(39.6)$ & $404(51.5)$ & $<0.001$ \\
\hline 2b. Discharge pack of emergency medications. & Yes/not applicable & $170(26.4)$ & $573(73.6)$ & $<0.001$ \\
\hline $2 a$ and $2 b$ & Yes & $133(20.6)$ & $352(45.3)$ & $<0.001$ \\
\hline \multicolumn{5}{|l|}{ 3. Assess smoking status and willingness to quit. } \\
\hline & $\begin{array}{l}\text { Smoker but cessation } \\
\text { not discussed }\end{array}$ & $124(23.4)$ & $102(14.4)$ & $<0.001$ \\
\hline & $\begin{array}{l}\text { Smoker/ex-smoker and } \\
\text { cessation discussed }\end{array}$ & $68(12.8)$ & $173(24.4)$ & \\
\hline & $\begin{array}{l}\text { Never smoker/ex- } \\
\text { smoker }\end{array}$ & $338(63.8)$ & $435(61.3)$ & \\
\hline \multicolumn{5}{|c|}{ 4. Assess for suitability of pulmonary rehabilitation prior to discharge. } \\
\hline & $\begin{array}{l}\text { Yes/completed rehab } \\
\text { previously/declined or } \\
\text { not applicable }\end{array}$ & $172(26.4)$ & $407(51.6)$ & $<0.001$ \\
\hline \multicolumn{5}{|c|}{ 5. Organise community follow-up within 2 weeks of discharge from hospital. } \\
\hline & $\begin{array}{l}\text { Yes, declined or not } \\
\text { applicable }\end{array}$ & $356(54.9)$ & $555(70.3)$ & $<0.001$ \\
\hline
\end{tabular}

AECOPD, acute exacerbation of chronic obstructive pulmonary disease.

that staff had insufficient time to change processes of care reliably.

\section{Strengths and limitations}

Due to the timing of care bundle implementation nationally, it was not possible to undertake a randomised controlled trial to evaluate the impact of care bundles. While site recruitment was facilitated through several routes, it is possible that the participating sites were those with a respiratory team who had an interest in care bundles or research activity related to COPD. Thus, they may have demonstrated 'better' performance with regard to COPD care and QI than average, introducing recruitment bias to the study.

Conducting research in the context of acute admissions posed challenges to patient recruitment and data collection, particularly in terms of data availability and completeness. Additional limitations of the study include potential reporting bias as some sites were unable to provide useful level 1 and 2 data. There were also differences in patient populations at different sites and implementation sites had higher baseline COPD readmission rates, and these factors could possibly have influenced the outcomes of the study.
With regard to the finding of a greater reduction in ED attendances in implementation sites than in comparator sites, we cannot exclude the possibility that this result arose by chance. A recent systematic review found only one study that considered the impact of COPD care bundles on ED attendances and found no effect. $^{12}$

These findings benefit from the pragmatic study design and robust analysis of detailed data from an extensive range of hospitals in England and Wales, enabling assessment of the delivery of care bundles in a 'real-life' care delivery context. Using a mixed-methods approach has ensured both the perception and practice of implementation have been evaluated. The findings are not necessarily due to lack of effectiveness of COPD bundles; rather they are likely due to lack of effective implementation of COPD bundles. This does not limit the importance of the results but highlights the 'real life' challenge of incorporating improvement science methods alongside reliable implementation of standard processes.

Future research should aim to address the temporality of QI practices through a longitudinal study offering greater and indepth analysis of the QI life cycle. Closer monitoring of implementation reliability would also be 
beneficial, ensuring that any observed lack of effect is related to the efficacy of the intervention rather than failure of implementation. The fields of improvement science and implementation research offer insights into more effective ways of facilitating QI in the NHS. ${ }^{25}$ Using these to ensure that QI efforts are as evidence-based as the best practice they seek to implement would enable future QI projects to make changes in the most effective way.

In summary, COPD care bundles had little impact on patient outcomes but appeared to be associated with a reduced number of subsequent ED attendances. The distinction between bundle implementation and standard care was not clearly defined and fidelity with care bundle delivery was generally very low, although care bundles had some impact on improving process reliability. These findings are unsurprising given the low level of bundle uptake in implementation sites, and it remains to be determined if COPD care bundles affect patient care and outcomes when they are effectively implemented. Healthcare professionals value care bundles, but despite the simplicity of the approach they are complex to implement, and clinicians require support to successfully change care pathways.

Acknowledgements This research has been conducted independently by the University of Bristol in partnership with staff from the University of the West of England, North Bristol NHS Trust and the British Thoracic Society. We thank all the patients, carers and healthcare practitioners who have contributed to the study to date, particularly our patient and public involvement panel who have helped define and guide the research. We are also grateful for the advice and support of our Study Steering Committee.

Contributors MC, SJ, AS, WH, CM, JB, JC and SP were responsible for drafting the original study protocol and securing funding for the research. All authors contributed to the delivery of the study and analysis and interpretation of data. KM and AK collected data from sites. SJ led on patient and public involvement. KM and SP wrote the original draft of the manuscript. All authors contributed to and approved the final manuscript on behalf of the wider research team.

Funding This work was supported by the National Institute for Health Research (NIHR) Health Services and Delivery Research (HS\&DR) Programme, project number $12 / 130 / 53$. The research was hosted by NHS Bristol and was designed and delivered in partnership with the Bristol Randomised Trials Collaboration (BRTC), a UK Clinical Research Collaboration (UKCRC) registered Clinical Trials Unit (CTU). The views expressed are those of the authors and not necessarily of the NHS, the NIHR or the Department of Health and Social Care.

Competing interests SP is a general practitioner, and JB and JC are hospital consultants working in the fields of emergency care and respiratory medicine, respectively. All have endeavoured to ensure that their input to the research has not been biased by their own clinical practice. JC worked with colleagues at the British Thoracic Society to design and evaluate care bundles as an intervention to improve outcomes in a number of different respiratory conditions, including chronic obstructive pulmonary disease, pneumonia and asthma. SP is a member of the National Institute for Health Research (NIHR) Health Services and Delivery Research Researcher-led Panel, from 2017 to date. WH is a member of the NIHR Health Technology Assessment Clinical Trials Board. SJ runs an independent consultancy for public and charitable sector clients, providing strategy and organisation development, leadership coaching and facilitation.

Patient consent for publication Not required.

Ethics approval Ethical approval was given by the South West (Frenchay) Research Ethics Committee (study reference 14/SW/1057).

Provenance and peer review Not commissioned; externally peer reviewed.

Data availlability statement All data relevant to the study are included in the article or uploaded as supplementary information.

Open access This is an open access article distributed in accordance with the Creative Commons Attribution 4.0 Unported (CC BY 4.0) license, which permits others to copy, redistribute, remix, transform and build upon this work for any purpose, provided the original work is properly cited, a link to the licence is given, and indication of whether changes were made. See: https://creativecommons.org/ licenses/by/4.0/.

\section{REFERENCES}

1. British Lung Foundation. The battle for Breath- the impact of lung disease in the UK. London: British Lung Foundation, 2016.

2. Mathers CD, Loncar D. Projections of global mortality and burden of disease from 2002 to 2030. PLoS Med 2006;3:e442-224.

3. Stone RA, Lowe D, Holzhauer-Barrie J. National COPD audit programme secondary care clinical audit COPD: who cares matters. London: Royal College of Physicians, 2015: 1-97.

4. Kayyali R, Odeh B, Frerichs I, et al. COPD care delivery pathways in five European Union countries: mapping and health care professionals' perceptions. Int J Chron Obstruct Pulmon Dis 2016;11:2831-8.

5. Price LC, Lowe D, Hosker HSR, et al. UK national COPD audit 2003: impact of hospital resources and organisation of care on patient outcome following admission for acute COPD exacerbation. Thorax 2006;61:837-42.

6. ResarR, GriffinFA, HaradenC. Using care bundles to improve health Care quality. IHI innovation series white paper. Cambridge, Massachusetts: Institute for Healthcare Improvement, 2012.

7. Calvert J, Lim WS, Rodrigo C, Turner A, Welham S. The British Thoracic Society pilot care bundle project: a care Bundles-Based approach to improving standards of care in chronic obstructive pulmonary disease. Vol. 6 No.4.British Thoracic Society Reports: 2014.

8. Robb E, Jarman B, Suntharalingam G, et al. Using care bundles to reduce in-hospital mortality: quantitative survey. BMJ 2010;340:c1234.

9. Hopkinson NS, Englebretsen C, Cooley N, et al. P100 designing and implementing a COPD discharge care bundle. Thorax 2011;66(Suppl 4) .

10. McCarthy C, Brennan J, Brown L, et al. Use of a care bundle in the emergency department for acute exacerbations of chronic obstructive pulmonary disease: a feasibility study. Int J Chron Obstruct Pulmon Dis 2013;8:605-11.

11. Zafar MA, Panos RJ, Ko J, et al. Reliable adherence to a COPD care bundle mitigates system-level failures and reduces COPD readmissions: a system redesign using improvement science. BMJ Qual Saf 2017;26:908-18.

12. Ospina MB, Mrklas K, Deuchar L, et al. A systematic review of the effectiveness of discharge care bundles for patients with COPD. Thorax 2017;72.

13. Laverty AA, Elkin SL, Watt HC, et al. Impact of a COPD discharge care bundle on readmissions following admission with acute exacerbation: interrupted time series analysis. PLOS ONE 2015;10:e0116187.

14. Turner AM, Lim WS, Rodrigo C, et al. A care-bundles approach to improving standard of care in AECOPD admissions: results of a National Project. Thorax 2015;70:992-4.

15. Chalder MJE, Wright CL, Morton KJP, et al. Study protocol for an evaluation of the effectiveness of 'care bundles' as a means of improving hospital care and reducing hospital readmission for patients with chronic obstructive pulmonary disease (COPD). BMC Pulm Med 2016;16.

16 NVivo qualitative data analysis Software; QSR International Pty Ltd. Version 10. 2012

17. Miles M, Huberman A. Qualitative data analysis: an expanded sourcebook. Thousand Oaks, California: Sage, 2004.

18. Geue C, Lewsey J, Lorgelly P, et al. Spoilt for choice: implications of using alternative methods of costing Hospital Episode Statistics. Health Econ 2012;21:1201-16.

19. Health and Social Care DO. NHS reference costs 2014-2015. Available: https://www.gov.uk/government/publications/nhsreference-costs-2014-to-2015/ [Accessed 30 January 2019].

20. Joint Formulary Committee. British National formulary. 70th ed. London: BMJ Group and Pharmaceutical Press, 2015.

21. PSSRU. Unit costs of health and social care, 2014. Available: http:// www.pssru.ac.uk/project-pages/unit-costs/2014/ [Accessed 30 January 2019].

22. Morton KJP, MacNeill SJ, Sanderson E, et al. An evaluation of 'care bundles' as a means of improving hospital care for patients with Chronic Obstructive Pulmonary Disease (COPD): results of a multi-site study in the United Kingdom. NIHR Health Serv Deliv Res 2019; in press. 
23. Lennox L, Green S, Howe C, et al. Identifying the challenges and facilitators of implementing a COPD care bundle. BMJ Open Respir Res 2014;1:e000035.

24. Dixon-Woods M, Leslie M, Tarrant C, et al. Explaining matching Michigan: an ethnographic study of a patient safety program. Implement Sci 2013;8.
25. The Health Foundation. Evidence scan: improvement science. Available: http://www.health.org.uk/sites/health/files/ ImprovementScience.pdf [Accessed 30 January 2019]. 